\title{
Continuous Frequency Tuning with near Constant Output Power in Coupled Y-Branched Terahertz Quantum Cascade Lasers with Photonic Lattice
}

Iman Kundu,*(-) Paul Dean, Alexander Valavanis, Joshua R. Freeman, Mark C. Rosamond, Lianhe Li, Yingjun Han, Edmund H. Linfield, and A. Giles Davies

School of Electronic and Electrical Engineering, University of Leeds, Woodhouse Lane, Leeds, LS2 9JT, United Kingdom

Supporting Information

ABSTRACT: We demonstrate continuous frequency tuning in terahertz quantum cascade lasers with double metal waveguides using a Y-branched coupler. Two THz QCLs placed side-by-side couple by evanescent fields across the air gap between them. Each QCL waveguide comprises a $48-\mu$ m-wide coupler and S-bend section, which are connected to an $88-\mu$ m-wide Y-branch through an impedance matching tapered section. Photonic lattices are patterned on top of the coupler section in each QCL using focused ion-beam milling to control the spectral characteristics. The waveguide design used for individual QCL sections is optimized using finite element modeling and the spectral

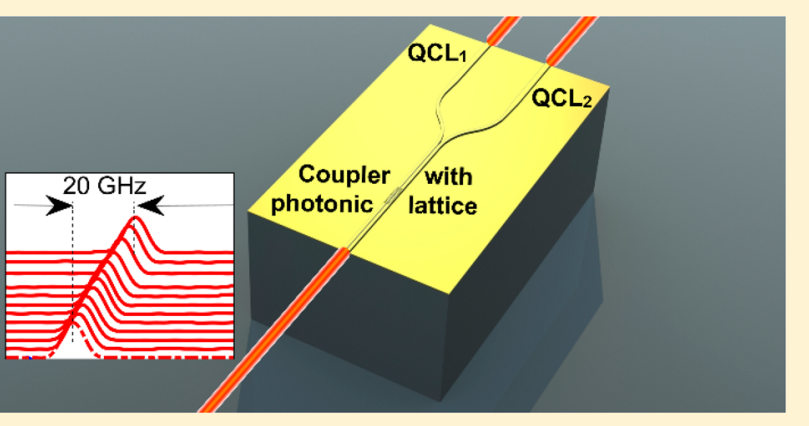
characteristics are modeled using a transfer matrix model.

Continuous frequency tuning of $\sim 19 \mathrm{GHz}$ is demonstrated while maintaining an output power of $\sim 4.2-4.8 \mathrm{~mW}$ and a heat sink temperature of $50 \mathrm{~K}$. The tuning is controlled electrically through Stark shift and cavity pulling effects by driving both QCLs simultaneously and represents the widest electrically controlled continuous tuning performance from a THz QCL without significant change in output power.

KEYWORDS: terahertz, quantum cascade laser, frequency tuning, single mode, Vernier tuning

$\mathrm{T}$ erahertz-frequency quantum cascade lasers (THz QCLs) ${ }^{1}$ are compact sources of radiation in the $\mathrm{THz}$ frequency band of the electromagnetic spectrum. They are attractive sources for a range of applications including gas spectroscopy, ${ }^{2}$ imaging, ${ }^{3}$ and heterodyne radiometry, ${ }^{4}$ many of which require continuously frequency tunable, narrowband emission. However, conventional $\mathrm{THz}$ QCLs based on Fabry-Pérot resonators employing either semi-insulating surface plasmon or double metal (DM) plasmonic waveguides, ${ }^{5}$ offer only limited frequency tunability. ${ }^{6}$

Although both discrete (mode hopping) and continuous (mode hop-free) tuning in $\mathrm{THz}$ QCLs have been demonstrated over frequency ranges $\sim 80-250^{7-9}$ and $\sim 3-50$ $\mathrm{GHz},{ }^{10-14}$ respectively, most of the tuning control techniques require complex and slow mechanical ${ }^{9}$ or electromechanical components, ${ }^{8}$ fiber coupled light sources, ${ }^{13}$ or gas or dielectric condensation. ${ }^{10,14}$ Conversely, continuous fast electrical tuning in multicavity $\mathrm{THz}$ QCLs has to date been limited to a bandwidth of $\sim 3-7.5 \mathrm{GHz} .{ }^{11}$ A frequency tuning of $30 \mathrm{GHz}$ has been reported using photonic crystals $(\mathrm{PhC})$ by changing the drive current across the dynamic operating range of the $\mathrm{THz} \mathrm{QCL}$, but this also results in a variation of the output power of $>80 \%$. ${ }^{15}$ On the other hand, wideband fast electrical tuning from monolithic semiconductor diode lasers have been realized using the Vernier effect. ${ }^{16}$ Such lasers comprise multiple sections, each of which support a comb of frequencies. Emission in such lasers is selectively favored at frequencies at which there is an alignment between the combs. Recently, such coupled-cavity schemes have emerged as versatile techniques to achieve single mode emission ${ }^{17}$ and frequency tuning ${ }^{12}$ in $\mathrm{THz}$ QCLs. However, all coupled-cavity $\mathrm{THz}$ QCLs to date have used semi-insulating surface plasmon waveguides, which result in lower operating temperatures in comparison with DM waveguides. Furthermore, in these studies, the THz QCLs were coupled in the end-fire direction, and so the mode selection was governed through multiplication of the frequency combs. An alternative Vernier scheme, however, exploits additive selection, where the supermode selection is performed through addition of the combs, which can lead to an improved suppression of the first side modes. ${ }^{18}$

In this work, we develop a new coupled-cavity scheme to extend the continuous tuning range and to improve suppression of first side modes in $\mathrm{THz}$ QCLs using the additive Vernier effect, in which two $\mathrm{DM} \mathrm{THz}$ QCLs are coupled laterally along the length of the cavity, similar to directional couplers. We also use finite defect site photonic lattices (PLs) to engineer the spectra in individual cavities. ${ }^{19}$

Received: February 23, 2018

Published: June 19, 2018 


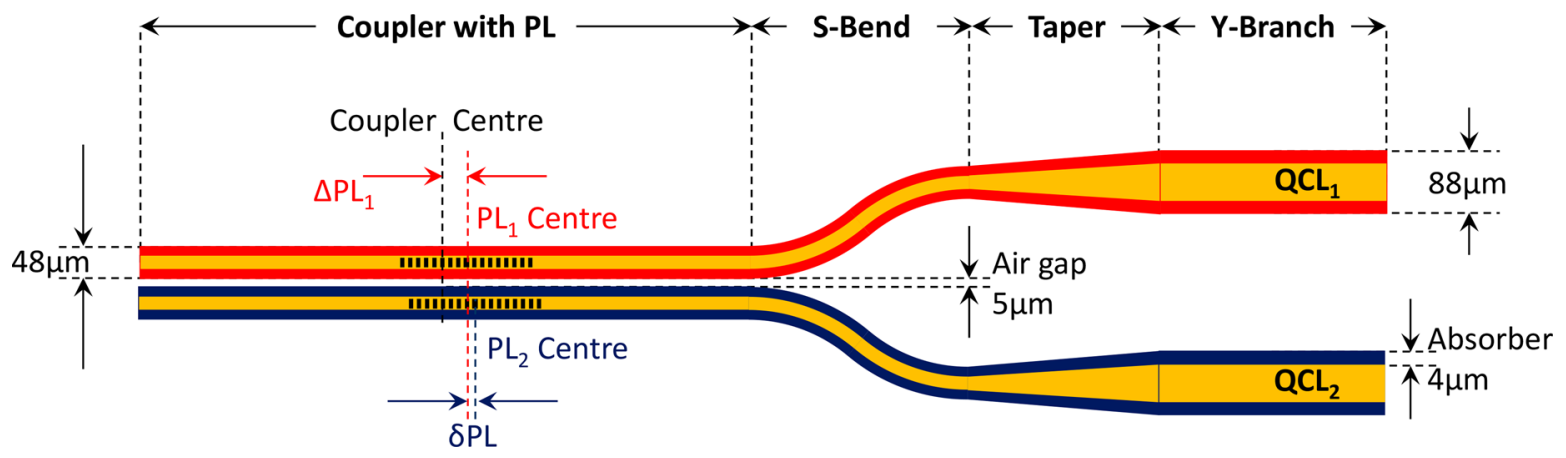

Figure 1. Illustration of Y-branched DM THz QCLs with PLs. Two optically coupled DM THz QCLs, $\mathrm{QCL}_{1}$ and $\mathrm{QCL}_{2}$, with integrated PLs: $\mathrm{PL}_{1}$ and $\mathrm{PL}_{2}$. The different waveguide sections and the longitudinal positions of PLs in the coupler sections are also illustrated.

Unlike a single cavity $\mathrm{THz}$ QCL incorporating a $\mathrm{PhC}$, distributed feedback lattice or PL, the coupled cavity approach used here allows frequency tuning while maintaining nearconstant output power. We report electrically controlled frequency tuning from such a coupled DM THz QCL with an integrated PL, and demonstrate continuous tuning of $\sim 19$ $\mathrm{GHz}$ at a heat sink temperature of $50 \mathrm{~K}$ with output power in the range of $\sim 4.2-4.8 \mathrm{~mW}$. The continuous tuning of the emission frequency from this device is achieved through a combination of a Stark shift of gain and cavity pulling effects.

\section{DEVICE DESIGN}

Our device comprises two DM THz QCLs $\left(\mathrm{QCL}_{1}\right.$ and $\left.\mathrm{QCL}_{2}\right)$, which are electrically isolated, but optically coupled in the lateral direction through a $5 \mu \mathrm{m}$ wide air gap, and is illustrated schematically in Figure 1. Each QCL consists of four waveguide sections: (1) a $48 \mu \mathrm{m}$ wide coupler with PL, which couples the electric field between two QCL ridges; (2) an $88 \mu \mathrm{m}$ wide "Y-branch" power amplifier to amplify the selected frequency; (3) an "S-shaped bend" that separates the amplifier sections spatially to avoid coupling between them; and (4) an impedance-matching taper section to match the wider amplifier to the S-bend section.

The narrow coupler section was used to excite a copropagating optical mode with the adjacent QCL ridge. A first-order, finite-defect-site $\mathrm{PL}$ was patterned in the coupler section in each QCL $\left(\mathrm{PL}_{1}\right.$ in $\mathrm{QCL}_{1}, \mathrm{PL}_{2}$ in $\left.\mathrm{QCL}_{2}\right)$ to achieve single mode emission. Unlike distributed feedback designs, where periodic perturbations are uniformly distributed along the length of the cavity, the PLs are comprised of only 15 defect sites on either side of a central $\pi$-phase shifted defect. A periodic modulation of the emission spectra is obtained from each PL defect site, where the periodicity of the modulation depends on the position of the defect. Moreover, the periodic arrangement of the defect sites results in a summation of all such spectral modulations arising from individual defect sites. $^{20,21}$ As such, the spectral performance and the frequency dependent transmission properties of a $\mathrm{THz}$ QCL with PL can be controlled by changing not only the PL periodicity and duty cycle, but also its relative position in the cavity. Such PL designs have been used previously in single cavity $\mathrm{THz}$ QCLs to demonstrate both continuous tuning and controlled mode hopping between discrete frequencies. ${ }^{19}$ In order to design a $\mathrm{PL}$ for continuous tuning, the design parameters are so selected such that transmission is favored only at a single frequency. However, to obtain discrete tuning the PL parameters are optimized such that transmission is supported at 2 or 3 frequencies. Frequency tuning (both continuous and discrete) is then realized by changing the drive current and bias across the QCL, resulting in a shift in peak gain due to the Stark shift. PL designs similar to those reported in ref 19 have been used here to enable continuous frequency tuning in $\mathrm{QCL}_{1}$ and discrete mode hopping in $\mathrm{QCL}_{2}$. In this way, the continuous tuning range in the coupled device can be increased significantly from that obtained from $\mathrm{QCL}_{1}$ alone by exploiting Vernier selection of the coupled cavity modes.

The narrow $48 \mu \mathrm{m}$ wide coupler section preserve the fundamental transverse mode, yet they result in lower output power. Additionally, they impose practical limitations on forming electrical connections to the devices. As such, $88 \mu \mathrm{m}$ wide waveguide sections were used in the Y-branches not only to allow electrical bonding to the devices, but also to increase the power output. These Y-branch sections are sufficiently spatially separated such that optical coupling is defined only by the coupler section. The coupler and Y-branch sections are connected using another $48 \mu \mathrm{m}$ wide $\mathrm{S}$-shaped bend section and an impedance-matching tapered section. A $4 \mu \mathrm{m}$ wide absorber was used at both edges of the two waveguides, along the entire length of the cavity, to suppress higher order transverse modes. 22

The design processes, described below, have three stages: (1) Optimizing the cavity lengths of the various waveguide sections, without the PLs, using finite element modeling (FEM) techniques. (2) The spectral characteristics of the device with the PLs was modeled using transfer matrices. (3) The field distribution in the coupled device with the PL was calculated using FEM.

Finite Element Modeling of Waveguide Sections and Y-Branched DM THz QCL. The fundamental optical modes in closely spaced waveguides couple through the evanescent field and split into a symmetric and an antisymmetric mode, each with different effective indices. Optical power in such coupled waveguides can be represented as a superposition of the symmetric and the antisymmetric modes. The degree of coupling between the two waveguides depends on geometric parameters, such as the width of the gap and length of the coupler, as well as the refractive index of the gap. The different propagation constants of the copropagating modes result in a transfer of the optical power between the coupled waveguides.

A complete transfer of optical power between the waveguides occurs after the so-called coupling length. The coupling length was calculated using the following expression: ${ }^{23}$

$$
L_{\mathrm{C}}=\frac{\pi}{\beta_{\mathrm{s}}-\beta_{\mathrm{a}}}
$$




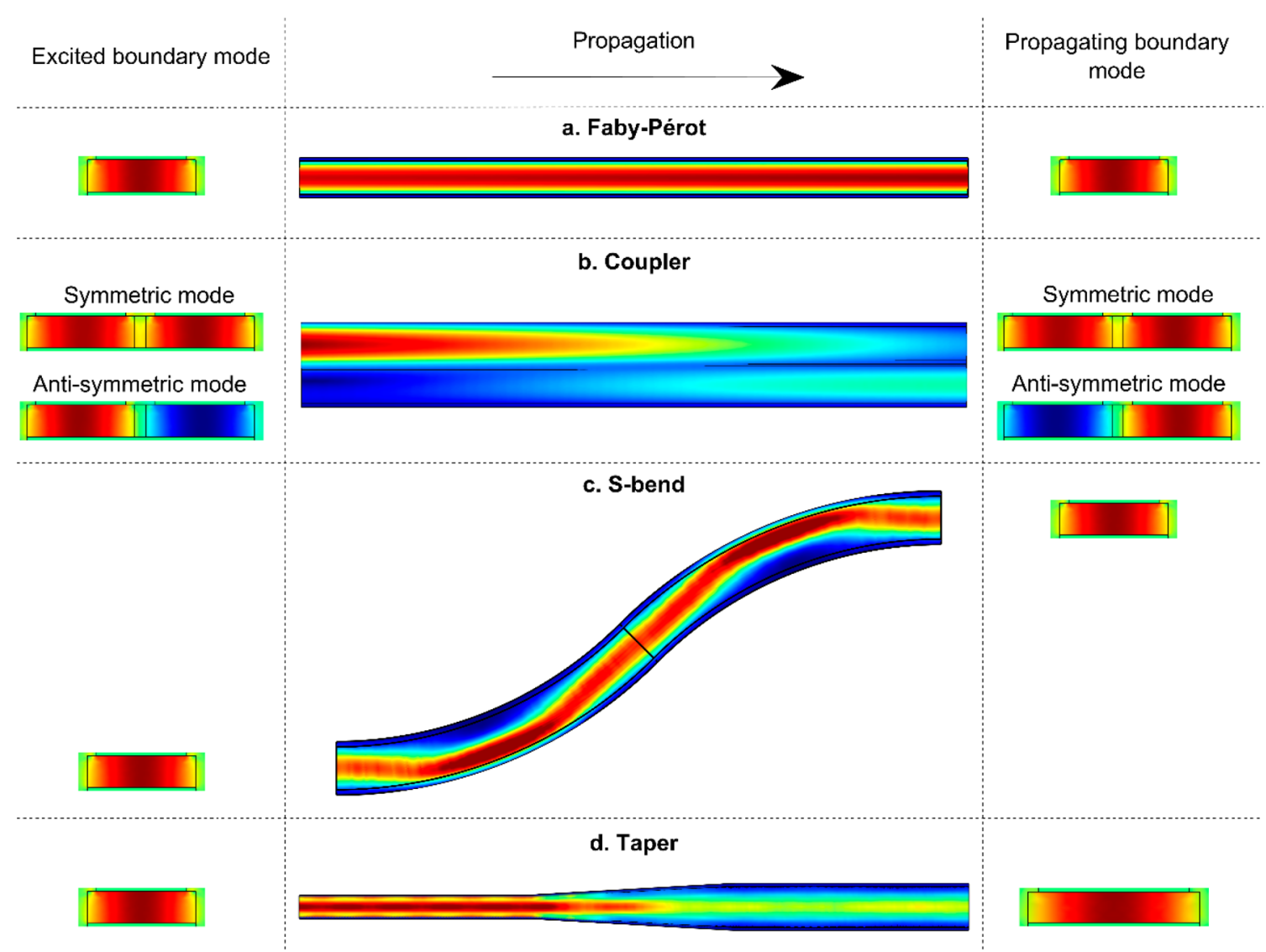

Figure 2. Simulated optical modes at waveguide boundaries and power distribution of the propagating electric field within individual waveguide components. (a) A $2.5 \mathrm{~mm}$ long standalone DM THz QCL. (b) Symmetric and antisymmetric modes in two $2.5 \mathrm{~mm}$ long QCLs coupled by an air gap. Coupling between the two QCLs is simulated as a superposition of the symmetric and the antisymmetric mode. (c) An S-bend section with radii of $340 \mu \mathrm{m}$. (d) A $350 \mu \mathrm{m}$ long taper section connecting a $48 \mu \mathrm{m}$ wide waveguide to a $88 \mu \mathrm{m}$ wide waveguide.

where $\beta_{\mathrm{s}}$ and $\beta_{\mathrm{a}}$ are propagation constants of the symmetric and the antisymmetric optical modes, which were calculated to be 262.983 and $262.268 \mathrm{rad} / \mathrm{mm}$, respectively.

In this study, a narrow air gap was used to couple optically, but isolate electrically, the two waveguides; an air gap of $5 \mu \mathrm{m}$ was selected to ensure sufficient optical coupling and electrical isolation between the waveguides. The coupling length for the $48-\mu \mathrm{m}$-wide waveguides coupled by a $5 \mu \mathrm{m}$ wide air gap was calculated to be $L_{C}=4.39 \mathrm{~mm}$. An equal distribution of optical power between the coupled waveguides was calculated to be $L_{\mathrm{C}} / 2=2.19 \mathrm{~mm}$.

The optical modes and the electric field coupling between the $\mathrm{THz}$ QCLs were simulated via FEM using a commercial software, COMSOL Multiphysics. A full three-dimensional model was used for the FEM analysis. Waveguide components were surrounded in an air atmosphere with a scattering boundary. Facet boundaries were modeled as ports and were used to excite the electric field propagating in the waveguide unidirectionally. In the first instance, propagation of the optical mode across each of the waveguide sections shown in Figure 1 was independently simulated using FEM (Figure 2). While the optical modes at both facets in a standalone cavity are identical (Figure 2a), the boundary modes in longitudinally coupled waveguides vary as a function of the cavity length. To enable a change in the polarity of the antisymmetric mode (Figure $2 b$ ) the length of the coupler section should be longer than $L_{C} / 2$ and was chosen to be $2.5 \mathrm{~mm}$ long. The ratio of optical power in each of the coupled waveguides, normalized to the total power, was calculated to be $0.43: 0.57$ in the $2.5 \mathrm{~mm}$ long coupler used in this study.

In order to reduce the threshold current of the individual QCLs, the design of the S-bend and the tapered sections were optimized to minimize their lengths, while preserving the fundamental transverse mode at the boundaries. To this end, S-bends consisting of two $45^{\circ}$ arcs with radii $r_{\mathrm{B}}=340 \mu \mathrm{m}$ were selected to be the shortest. In such S-bends, the electric field propagates using whispering gallery modes (Figure 2c). Similarly, a $350 \mu \mathrm{m}$ long tapered waveguide section was selected to match the impedance between the S-bends and the Y-branches (Figure 2d). The length of the Y-branches was chosen to be $1.1 \mathrm{~mm}$, such that the length of the entire device is $\sim 4.5 \mathrm{~mm}$.

The effective refractive index of the optical mode in the PL was also calculated using FEM (for further details see Supporting Information). The effective index of the mode at the metallized section was calculated to be $n_{\text {eff, }}=3.58$. A $3 \mu \mathrm{m}$ deep etch was used in the etched section of the PL. The refractive index contrast between the metallized and the etched section of the PL was calculated to be $\Delta n=0.59$.

Transfer Matrix Modeling of Y-Branched THz QCLs with PLs. The spectral behavior of the device with PLs added to the coupler sections was modeled using transfer matrices. The PL was designed using a transfer matrix model (see Supporting Information).

A transfer matrix describing the propagation of the electric field at each QCL along the various waveguide sections, 

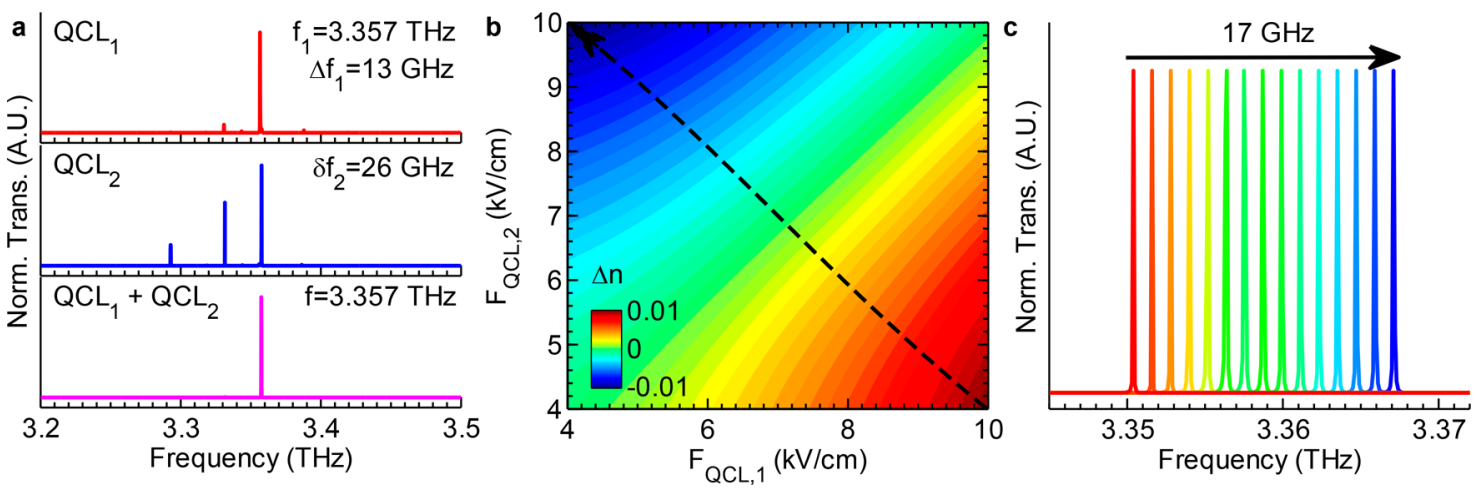

Figure 3. Frequency tuning from the coupled device simulated using transfer matrices. (a) Transmission peaks from $\mathrm{QCL}_{1}$ and $\mathrm{QCL} \mathrm{L}_{2}, \mathrm{when}$ operating independently, and when both QCLs are coupled. (b) Change in refractive index due to both Stark shift of gain and cavity pulling effects, simulated as a function of $F_{\mathrm{QCL}, 1}$ and $F_{\mathrm{QCL}, 2}$. A linear variation of the refractive index is illustrated using a dashed arrow. (c) Simulated continuous frequency tuning controlled through Stark shift and cavity pulling in the coupled device.

coupler, S-bends, taper, and Y-branched, was calculated. The transfer matrix of the PL and additional bending losses arising from the S-bends were also included in the transfer matrices. The transfer matrix of each QCL forming the Y-branched coupler were modeled as following:

$$
T_{n}=T_{\mathrm{C}, \mathrm{F}, n} \times T_{\mathrm{PL}} \times T_{\mathrm{C}, \mathrm{R}, n} \times T_{\mathrm{S}} \times T_{\mathrm{T}} \times T_{\mathrm{Y}}
$$

where $n=1,2$ for the two QCLs. $T_{\mathrm{C}, \mathrm{F}}$ and $T_{\mathrm{C}, \mathrm{R}}$ are transfer matrices for the coupler at either side of the PL, which is described with a transfer matrix $T_{\mathrm{PL}}$. The transfer matrix of the S-bend, taper, and Y-branches were modeled with transfer matrices $T_{\mathrm{S}}, T_{\mathrm{T}}$, and $T_{\mathrm{Y}}$, respectively. The coupling between the two QCLs were calculated using the following scattering matrix: ${ }^{24}$

$$
S=\left[\begin{array}{cc}
\tau & -j \kappa \\
-j \kappa & \tau
\end{array}\right]
$$

where $\kappa$ and $\tau$ are coupling and transmission coefficients in the coupler and vary as a function of the coupler length and $-j$ is the phase factor.

In order to increase the continuous frequency tuning range, the PL patterned on one of the two QCL ridges was designed to support continuous tuning while the other was designed to support controlled mode hopping between discrete frequencies, when operated independently. When operated simultaneously, this enables wideband and continuous frequency coverage with a single lasing mode, through a combination of mode hopping and fine continuous frequency tuning. As such, one of the PLs $\left(\mathrm{PL}_{1}\right.$ on $\left.\mathrm{QCL}_{1}\right)$ was designed to operate at only one frequency $\left(f_{1}\right)$ with a continuous frequency tuning $\left(\Delta f_{1}\right)$, when operating independently. The second $\mathrm{PL}\left(\mathrm{PL}_{2}\right.$ on $\left.\mathrm{QCL}_{2}\right)$ was designed to support mode hopping between widely spaced cavity modes, similar to that reported in ref 19 . We note that in such designs continuous tuning $\left(\Delta f_{2} \sim \Delta f_{1}\right)$ around each of the discrete modes is also possible. The inclusion of this modehope based tuning in $\mathrm{QCL}_{2}$ serves to increase the total spectral coverage of the coupled device through Vernier selection. To this end, $\mathrm{PL}_{2}$ was designed to have multiple resonances close to $f_{1} \pm \Delta f_{1}$ and to obtain discrete tuning such that the frequency separation between the mode hops is $\delta f_{2} \sim 2 \times \Delta f_{1}$, when operating independently. The emission frequency in the coupled device is selected through Vernier selection and phase matching conditions, when both cavities are operated simultaneously. The frequency tuning range can also be extended further through electrical control of the drive currents in both QCL ridges, and by exploiting the Stark shift of the gain in the QCL gain region as a function of the applied field, ${ }^{19}$ as well as cavity pulling in the coupled waveguides. ${ }^{11}$ As such, this design should enable continuous spectral coverage over a frequency range of up to $\delta f_{2}$ and result in an improvement in the side mode suppression ratio (SMSR) through the additive Vernier effect.

The Stark shift was calculated from the applied field $(F):^{25}$

$$
\Delta E_{\mathrm{S}}=e F\left(\left\langle\Psi_{2}|z| \Psi_{2}\right\rangle-\left\langle\Psi_{1}|z| \Psi_{1}\right\rangle\right)
$$

where $\Psi_{1}$ and $\Psi_{2}$ are the wave functions of the lower and upper laser levels calculated using a Schrödinger-Poisson solver and $e$ is the elementary charge. The Stark shift of the QCL material was calculated from the dynamic operating range of reference devices (applied field in the range $F=4-10$ $\mathrm{kV} / \mathrm{cm}$, or a change in the terminal voltage of $\Delta V=8.5 \mathrm{~V}$ ) to be $\Delta E_{\mathrm{S}}=2.64 \mathrm{meV}$. The Stark shift as a function of applied electric field across the QCL was also calculated to be $\mathrm{d} E_{\mathrm{S}} / \mathrm{d} V$ $=0.504 \mathrm{meV} / \mathrm{V}$ or $121 \mathrm{GHz} / \mathrm{V}$. The change in emission spectra due to Stark shift was studied from single mode reference QCLs fabricated from the same wafer. A change in emission frequency per unit voltage was calculated from the experimental data to be $\mathrm{d} f / \mathrm{d} V=0.5 \mathrm{GHz} / \mathrm{V}(\mathrm{d} \lambda / \mathrm{d} V=51 \mathrm{~nm} /$ $\mathrm{V})$. The variation of refractive index as a function of applied voltage across the device was calculated from the experimental data to be $\mathrm{d} n / \mathrm{d} V=0.0021 / \mathrm{V}$ using the relationship $(1 / n)(\mathrm{d} n /$ $\mathrm{d} V)=(1 / \lambda)(\mathrm{d} \lambda / \mathrm{d} V)$.

For simplicity, both PLs were designed with an identical lattice pitch $(\Lambda)$ and with 15 defect sites on either side of a central $\pi$-shift element. Initially, a PL located at the center of the coupler section and with an emission frequency of $\sim 3.36$ $\mathrm{THz}$ was chosen, to match the active region gain $\sim 3.3-3.6$ THz. The pitch of the PL was calculated to be $\Lambda=13.66 \mu \mathrm{m}$, for an aperture width of $L_{\mathrm{PL}, \mathrm{e}}=7.44 \mu \mathrm{m}$ and an etch depth of 3 $\mu \mathrm{m}$. The aperture of the central $\pi$-shift element was selected to be $L_{\mathrm{PL}, \mathrm{pi}}=2 \times L_{\mathrm{PL}, \mathrm{e}}$. Next, an algorithm was used to optimize the spectral characteristics of $\mathrm{PL}_{1}$ and $\mathrm{PL}_{2}$ by offsetting the location of the PLs with respect to the center of the coupler section iteratively in steps of $\Lambda / 4$ for each. By solving the transfer matrices for each iteration, to optimize continuous tuning an discrete mode hops respectively, an offset of $\mathrm{PL}_{1}$ from the center of the coupler by $\Delta \mathrm{PL}_{1}=6.5 \times \Lambda$, and an offset of $\mathrm{PL}_{2}$ from $\mathrm{PL}_{1}$ by $\delta \mathrm{PL}=0.5 \times \Lambda$ were found. $\mathrm{QCL}_{1}$ was simulated to lase at a frequency $f_{1}=3.357 \mathrm{THz}$. A 

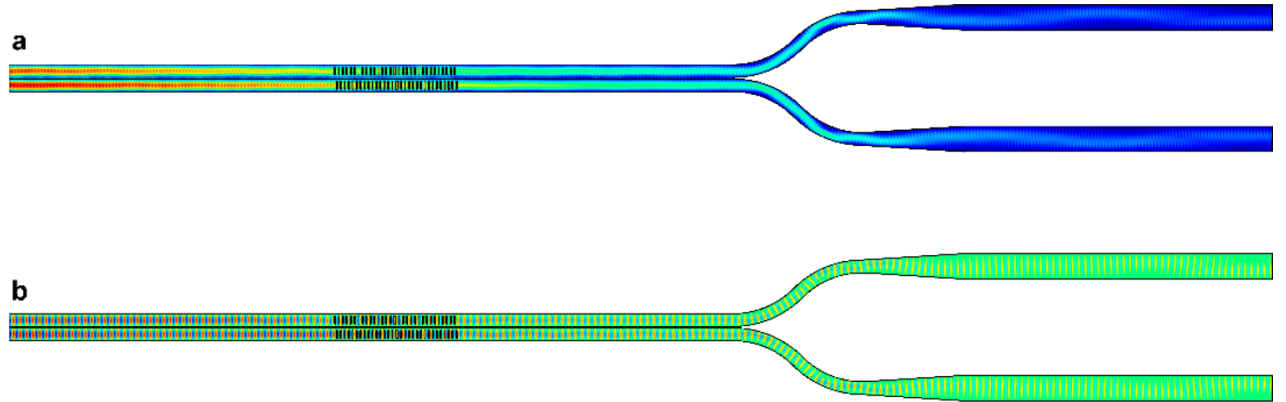

Figure 4. Simulated power distribution of the propagating electric field in the Y-branched DM THz QCL with PLs in the coupler section. (a) Power and (b) electric field in a $4.5 \mathrm{~mm}$ long device with a $2.5 \mathrm{~mm}$ long coupler and $1.1 \mathrm{~mm}$ long Y-branches.

continuous tuning range of $\Delta f_{1} \sim 13 \mathrm{GHz}$ (centered at $f_{1}$ ) was also simulated through Stark shift of the gain when the electric field across $\mathrm{QCL}_{1}$ was varied by $\Delta F_{\mathrm{QCL}, 1}=6 \mathrm{kV} / \mathrm{cm}$ and $\mathrm{QCL}_{2}$ was switched off (Figure 3a). Similarly, transmission peaks in $\mathrm{QCL}_{2}$ were simulated to be at 3.358 and 3.332 , a frequency separation of $\delta f_{2} \sim 26 \mathrm{GHz}$, with a smaller transmission peak at $3.293 \mathrm{THz}$. The emission frequency in $\mathrm{QCL}_{2}$ can be tuned between these frequencies through a similar Stark shift of the gain. The coupled device, comprising of $\mathrm{QCL}_{1}$ and $\mathrm{QCL}_{2}$ with the respective PLs was then simulated, producing a Vernier resonance at $3.357 \mathrm{THz}$.

The frequency tuning mechanism of the coupled device is through a change in the refractive index of the active region resulting from Stark shift of gain, together with cavity pulling effects. The frequency dependent gain in the QCL results in a dispersion of the real part of the refractive index arising through Kramer-Krönig relationship. ${ }^{26}$ This frequency dependent dispersion was used in conjunction with the Stark shift in ref 11 to tune the refractive index in multicavity QCLs through cavity pulling effects. A similar approach has been adopted here, where the gain in the coupled cavities are varied independently to change the refractive index in the device. The change in refractive index due to both Stark shift of gain and cavity pulling was simulated to be $-10.5 \times 10^{-3} \leq \Delta n \leq 10.5$ $\times 10^{-3}$, calculated by systematically varying the electric field in $\mathrm{QCL}_{1}\left(F_{\mathrm{QCL}, 1}\right)$ and $\mathrm{QCL}_{2}\left(F_{\mathrm{QCL}, 2}\right)$ in the range $4-10 \mathrm{kV} / \mathrm{cm}$ (Figure $3 \mathrm{~b}$ ). A continuous frequency tuning of $\sim 17 \mathrm{GHz}$, from 3.35 to $3.367 \mathrm{THz}$, was calculated by simulating a change in the refractive index for the coupled device (Figure $3 \mathrm{c}$ ). We note that the net gain in the coupled device is constant along the dashed line in Figure 3b.

Finite Element Model of Complete Device. Finally, electric field propagation in the complete device was simulated using FEM (Figure 4). The electric field in the coupled waveguide is reflected in the $\mathrm{PL}$ and changes the power distribution in the coupled waveguides. The ratio of the optical power in each of the coupled waveguides with the PL was calculated to be $0.47: 0.53$. Although the field propagation in the device was simulated to be due to the fundamental transverse mode, a spatial distortion of the power distribution is simulated in the Y-branches. This is attributed to the unidirectional propagation of the optical modes simulated in the model, which does not account for feedback from the facets. However, in a real device, such distortions and any higher order transverse modes would not be supported due to the higher losses resulting from the absorbers in the waveguide, as illustrated in Figure 1.

\section{THZ QCL GROWTH AND FABRICATION}

A THz QCL, based on the active region design reported in ref 27 , was rescaled to operate at $\sim 3.3-3.6 \mathrm{THz}$ and grown using molecular beam epitaxy in the $\mathrm{GaAs} / \mathrm{Al}_{0.18} \mathrm{Ga}_{0.82} \mathrm{As}$ material system. Growth started with a $250 \mathrm{~nm}$-thick GaAs buffer layer grown on a GaAs substrate, followed by a $300 \mathrm{~nm}$ thick $\mathrm{Al}_{0.50} \mathrm{Ga}_{0.50}$ As etch stop layer. A $700 \mathrm{~nm}$ thick $n$-doped GaAs layer, doped with $\mathrm{Si}$ at $2 \times 10^{18} \mathrm{~cm}^{-3}$, was grown next. This was followed by 115 repetitions of the active region layers structure. Alternating layers of $\mathrm{Al}_{0.18} \mathrm{Ga}_{0.82} \mathrm{As} / \mathrm{GaAs}$ forming the active region stack were then grown in a sequence starting from the injection barrier: 3.8/14.2/2.9/16.3/2.9/6.8/2.9/8.2/ 2.9/9.1/2.9/11.3/1.9/12.9/1/12.6/0.5/10.8 nm (barriers in italics). The $16.3 \mathrm{~nm}$ wide well (underlined) was $n$-doped with $\mathrm{Si}$ at $3 \times 10^{16} \mathrm{~cm}^{-3}$. Growth concluded with a $50 \mathrm{~nm}$-thick $n$ doped GaAs layer, doped with $\mathrm{Si}$ at $5 \times 10^{18} \mathrm{~cm}^{-3}$.

DM THz QCL devices were then fabricated. A $10 \mathrm{~nm}$ thick film of Ti and a $500 \mathrm{~nm}$ thick film of Au were deposited on top of $\mathrm{THz}$ QCL chips and $n^{+}$-doped receptor substrates using electron beam evaporation. The THz QCL and the $n^{+}$-doped receptor chips were then flipchip bonded using thermocompression wafer bonding technique. The substrate of the $\mathrm{THz}$ QCL was mechanically lapped. The $300 \mathrm{~nm}$ thick etch stop layer was etched using HF and the $700 \mathrm{~nm}$ thick highly doped epilayer was then etched away using an etchant solution of $\mathrm{H}_{2} \mathrm{SO}_{4} / \mathrm{H}_{2} \mathrm{O}_{2} / \mathrm{H}_{2} \mathrm{O}$. A $10 \mathrm{~nm}$ thick Ti and $200 \mathrm{~nm}$ thick $\mathrm{Au}$ were deposited using thermal evaporation forming the cladding metal. Ridge waveguides were defined using inductively coupled plasma reaction ion etching using $\mathrm{BCl}_{3} / \mathrm{Cl}_{2} / \mathrm{N}_{2}$ to achieve vertical sidewalls. The $n^{+}$-doped receptor substrate was thinned to a thickness of $\sim 200 \mu \mathrm{m}$ using wet chemical etching. Individual devices were cleaved and soldered onto $\mathrm{Cu}$ receptors. Packaged devices were patterned with a FEI Helios G4 CX DualBeam focused ion beam to form the PLs.

\section{CHARACTERIZATION}

$\mathrm{THz}$ QCLs were characterized in a Janis ST-100 continuous flow helium-cooled cryostat in pulsed mode, and were electrically driven with $10 \mathrm{kHz}(2 \mu$ s wide $)$ quasi-direct current pulses. Average power was measured using a Ge:Ga photodetector, and calibrated using a Thomas Keating absolute $\mathrm{THz}$ power meter. Spectra were acquired using a Bruker Fourier transform infrared spectrometer with a resolution of $7.5 \mathrm{GHz}$. Although the dynamic operating range of the QCL material used in this study has been measured from references devices to be in the range $\sim 0.35-2$ A (current density in the range $180-580 \mathrm{~A} / \mathrm{cm}^{2}$ ), the maximum drive currents in $\mathrm{QCL}_{1}$ and $\mathrm{QCL}_{2}$ were restricted 
to $\sim 1.6 \mathrm{~A}$ to avoid damage to the device. LIV characteristics were also measured before the PL patterning. In this case, peak output powers of $6 \mathrm{~mW}$ and $4.1 \mathrm{~mW}$ were measured from the two Y-branches. The ratio of output power, normalized to the total power, was thus calculated to be 0.41:0.59 and is in close agreement with the simulated value of 0.43:0.57.

\section{RESULTS}

Light-current-voltage (LIV) characteristics in pulsed mode were measured from the facets of both the coupler and Ybranch sections when $\mathrm{QCL}_{1}$ and $\mathrm{QCL}_{2}$ were operated independently (i.e., with the other waveguide unbiased), at a heat sink temperature $T_{\mathrm{HS}}=10 \mathrm{~K}$ (Figure 5). A peak output

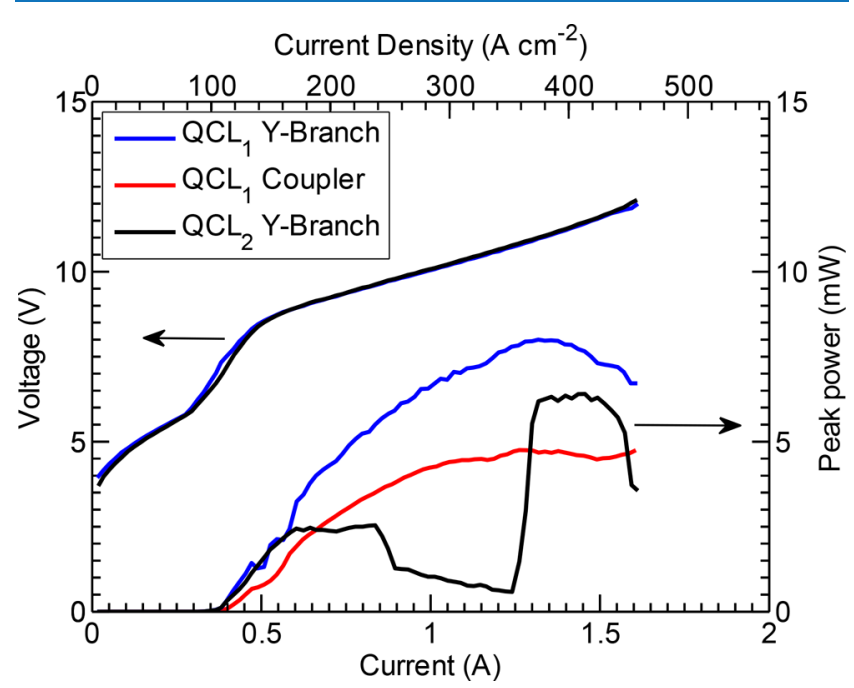

Figure 5. Experimentally recorded LIV (at a heat sink temperature of $10 \mathrm{~K}$ ). Peak output power from $\mathrm{QCL}_{1}$ (at both facets at the Y-branch and at the coupler), $\mathrm{QCL}_{2}$ (Y-branch), and $I-V$ characteristics recorded from $\mathrm{QCL}_{1}$ and $\mathrm{QCL}_{2}$ are shown as a function of drive current amplitude and current density.

power of $4.8 \mathrm{~mW}$ was measured from the coupler section facet for both QCLs, while peak output powers of 8 and $6.5 \mathrm{~mW}$ were recorded from the Y-branch facets of $\mathrm{QCL}_{1}$ and $\mathrm{QCL}_{2}$, respectively. This asymmetry of the output powers from the Ybranch sections is indicative of the coupling of electric field between $\mathrm{QCL}_{1}$ and $\mathrm{QCL}_{2}$. The ratio of output power from the Y-branch sections of each QCL, normalized to the total power, was calculated to be $0.45: 0.55$. The slight discrepancy between the experimental and simulated value of $0.47: 0.53$ could be due to collection inefficiency from the facet arising due to the subwavelength confinement of the optical modes in the DM QCLs. The attenuation of emitted power from $\mathrm{QCL}_{2}$ at drive currents $I_{\mathrm{QCL}, 2}=0.65-1.25 \mathrm{~A}$ is due to atmospheric absorption, verified by the spectral characterization below. Nevertheless, the variation in the measured power between the facets and between the two Y-branches is indicative of optical coupling and the resulting asymmetry in the power distribution in the device.

Spectra were recorded from the coupler section facet with each QCL electrically driven independently at $T_{\mathrm{HS}}=10 \mathrm{~K}$, and the other waveguide unbiased. Figure 6 a shows the spectra from $\mathrm{QCL}_{1}$, displaying single mode emission centered at $\sim 3.355 \mathrm{THz}$ and a continuous tuning in the range 3.353$3.362 \mathrm{THz}$ as the drive current was varied in the range $I_{\mathrm{QCL}, 1}=$ 0.5-1.6 A, corresponding to a change in the terminal voltage

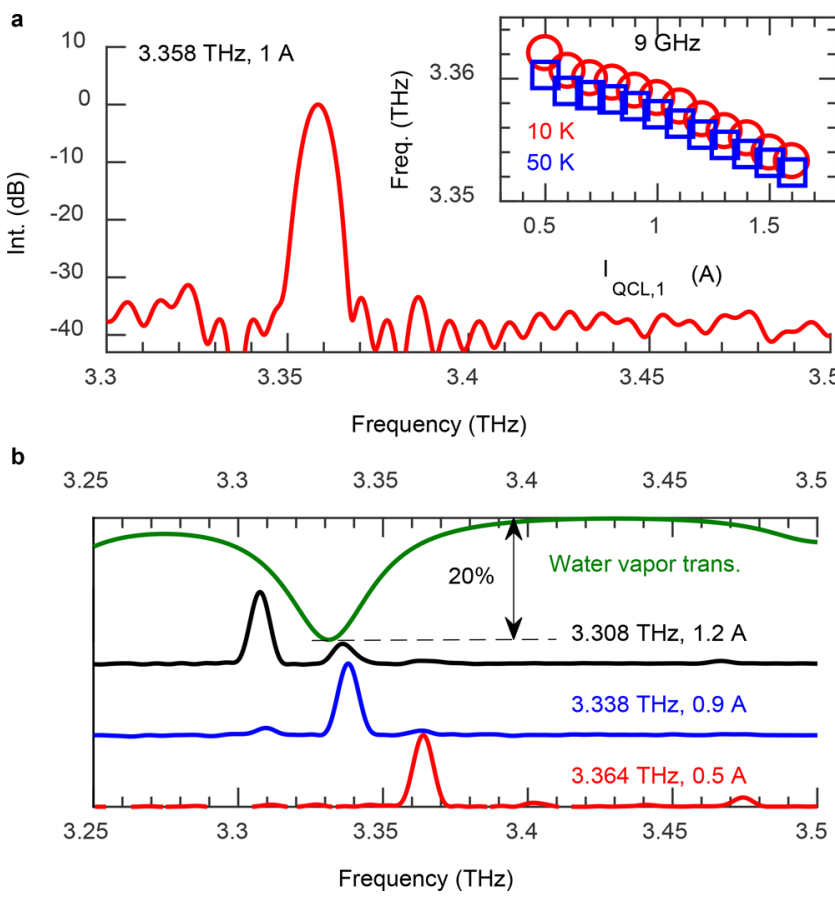

Figure 6. Experimentally recorded spectra from $\mathrm{QCL}_{1}$ and $\mathrm{QCL}_{2}$ when driven independently. (a) Emission frequency from $\mathrm{QCL}_{1}$ with $\mathrm{QCL}_{2}$ unbiased, at $T_{\mathrm{HS}}=10 \mathrm{~K}$. Inset: Peak emission frequency as a function of drive current at $T_{\mathrm{HS}}=10$ and $50 \mathrm{~K}$. (b) Normalized emission frequency from $\mathrm{QCL}_{2}\left(\mathrm{QCL}_{1}\right.$ is unbiased $)$ at $T_{\mathrm{HS}}=10 \mathrm{~K}$ and the normalized transmission spectra of water vapor.

of $\Delta V_{\mathrm{QCL}, 1}=6 \mathrm{~V}\left(\Delta F_{\mathrm{QCL}, 1}=4.3 \mathrm{kV} / \mathrm{cm}\right)$. The emission frequencies agree well with the simulations (Figure $3 \mathrm{a}$ ). Although continuous tuning in the range of $\Delta f_{1}=13 \mathrm{GHz}$ was simulated, the experimentally observed value of $\Delta f_{1}=9 \mathrm{GHz}$ was due to the lower drive current supplied to $\mathrm{QCL}_{1}$. A similar continuous tuning range of $\sim 8 \mathrm{GHz}$, spanning frequencies $3.352-3.36 \mathrm{THz}$, was also recorded at $T_{\mathrm{HS}}=50 \mathrm{~K}$ (Figure $6 \mathrm{a}$, inset).

Discrete mode hops between frequencies 3.364, 3.338, and $3.308 \mathrm{THz}$ was observed from $\mathrm{QCL}_{2}$ as a function of the drive current, $I_{\mathrm{QCL}, 2}=0.5-1.5 \mathrm{~A}$ (Figure $6 \mathrm{~b}$ ). The frequency separation of $\delta f_{2}=27 \mathrm{GHz}$ between the mode hops agrees well with the simulated value of $\delta f_{2}=26 \mathrm{GHz}$. Additionally, the attenuation of emitted power from $\mathrm{QCL}_{2}$ (Figure 5) was studied by comparing the emission frequencies with water vapor. ${ }^{28}$ The decrease in the measured power for $\mathrm{QCL}_{2}$ at current amplitudes $I_{\mathrm{QCL}, 2}=0.80-1.10$ A (Figure 5) coincided with an emission frequency of $3.338 \mathrm{THz}$, where the water vapor absorption is $\sim 20 \%$ higher than all other emission frequencies from the device. As such, the decrease in the measured power at these drive currents is attributed to atmospheric absorption at that emission frequency.

Spectra and output power from the device were also recorded with $\mathrm{QCL}_{1}$ and $\mathrm{QCL}_{2}$ driven simultaneously at different drive current amplitudes for heat sink temperatures $T_{\mathrm{HS}}=10$ and $50 \mathrm{~K}$ (Figure 7 ). To prevent damage to the device, initially the total drive current supplied to the composite device was restricted to be $\sim 2.5 \mathrm{~A}$. As such, drive currents in both QCL ridges were varied within the range: $I_{\mathrm{QCL}, 1}=0-1 \mathrm{~A}$ and $I_{\mathrm{QCL}, 2}=0.5-1.5 \mathrm{~A}$. Discrete tuning between frequencies $\sim 3.36,3.34$, and $3.31 \mathrm{THz}$ was recorded at $T_{\mathrm{HS}}=10 \mathrm{~K}$ (Figure $\left.7 \mathrm{a}\right)$, when $I_{\mathrm{QCL}, 2}$ was varied in the range 
a
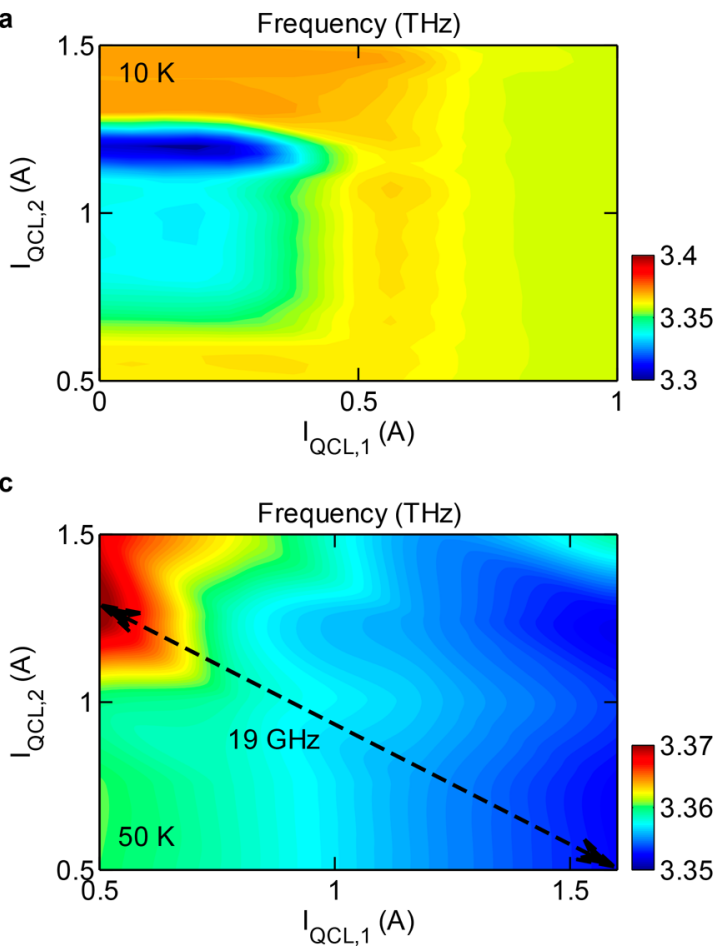

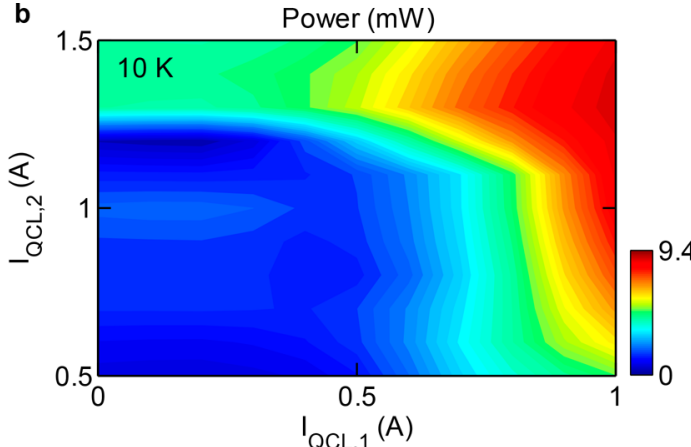

d

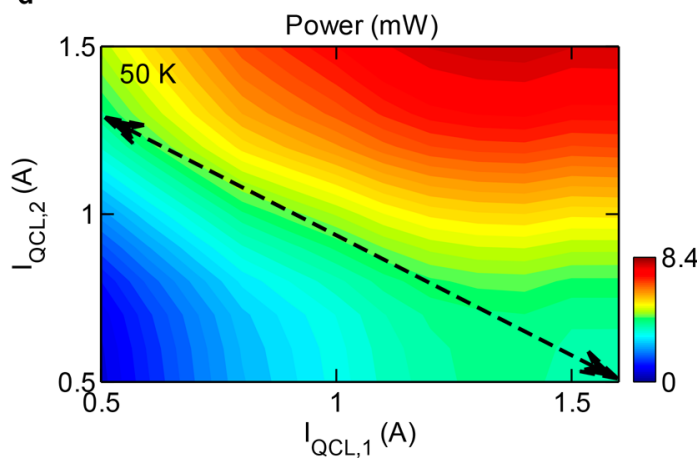

Figure 7. Experimentally recorded variation in emission frequency and output power as a function of drive current amplitudes in $\mathrm{QCL}_{1}$ and $\mathrm{QCL}_{2}$. Change in (a) emission frequency and (b) output power at $T_{\mathrm{HS}}=10 \mathrm{~K}$. (c) A continuous tuning of emission frequency, and (d) the corresponding change in output power at $T_{\mathrm{HS}}=50 \mathrm{~K}$. A linear change in frequency and the corresponding output power is illustrated using arrows.

0.5-1.5 A and $\mathrm{QCL}_{1}$ was below threshold. At these drive conditions, the maximum output was recorded to be $4.8 \mathrm{~mW}$ (Figure $7 \mathrm{~b}$ ), and an attenuation of emitted power was recorded at $I_{\mathrm{QCL}, 2}=1-1.25 \mathrm{~A}$, similar to that observed in Figure 5. However, a mode hop-free continuous single-mode tuning of $\sim 11 \mathrm{GHz}$ between $\sim 3.359-3.370 \mathrm{THz}$ was recorded when $\mathrm{QCL}_{1}$ was driven above threshold (at current amplitudes $I_{\mathrm{QCL}, 1}$ $=0.5-1 \mathrm{~A}$ ) and $I_{\mathrm{QCL}, 2}=0.5-1.5 \mathrm{~A}$. This continuous tuning is due to the Vernier frequency selection in the coupled device, and also coincided with an increase in the output power. Furthermore, a maximum peak power of $\sim 9.4 \mathrm{~mW}$ with no atmospheric absorption of output power was observed at these drive conditions. We also note that the continuous tuning range recorded here is larger than that obtained from $\mathrm{QCL}_{1}$ alone $(9 \mathrm{GHz})$ and is due to the Stark shift of the gain and cavity pulling effect.

Increasing the heat sink temperature changes the refractive index contrast in the PL. ${ }^{19}$ In order to increase the continuous tuning range, the total drive current supplied to the composite device was increased to $\sim 3 \mathrm{~A}$. Through these changes an emission frequency centered at $\sim 3.36 \mathrm{THz}$ was recorded at $T_{\mathrm{HS}}=50 \mathrm{~K}$, as drive currents in both QCL ridges were varied in the range $0.5-1.6 \mathrm{~A}$. A continuous tuning of the emission frequency is plotted in Figure $7 \mathrm{c}$. A continuous tuning range of $8 \mathrm{GHz}(\sim 3.352-3.360 \mathrm{THz})$ was recorded when $I_{\mathrm{QCL}, 1}$ was varied in the range $0.5-1.5 \mathrm{~A}$, and $I_{\mathrm{QCL}, 2}<1 \mathrm{~A}$. However, this increased to $19 \mathrm{GHz}$ (from $3.352 \mathrm{THz}$ to $3.371 \mathrm{THz}$ ) when $I_{\mathrm{QCL}, 2}>1.2 \mathrm{~A}$ and $I_{\mathrm{QCL}, 1}=0.5-1.6 \mathrm{~A}$. The experimentally recorded tuning range agrees well with the simulated tuning range of $17 \mathrm{GHz}$. A linear variation of the emission frequency is realized through a variation of $I_{\mathrm{QCL}, 1}$ and $I_{\mathrm{QCL}, 2}$ (Figure $7 \mathrm{c}$, arrow), and agrees well with the simulated linear change in refractive index.
Additionally, the output power varies only slightly in the range $\sim 4.2-4.8 \mathrm{~mW}$ (Figure $7 \mathrm{~d}$, arrow) when the linear shift in emission frequency is recorded, and is due to the constant net gain in the coupled device at these drive conditions. The maximum output power from the device was recorded to be $\sim 8.4 \mathrm{~mW}$, at $T_{\mathrm{HS}}=50 \mathrm{~K}$ (Figure $\left.7 \mathrm{~d}\right)$.

To assess the complete spectral characteristics of the device, spectra were similarly recorded for a range of currents, $I_{\mathrm{QCL}, 1}$ and $I_{\mathrm{QCL}, 2}$, and at $T_{\mathrm{HS}}=10-90 \mathrm{~K}$ (Figure 8). Emission frequencies similar to those in Figure $7 \mathrm{a}, \mathrm{c}$ were recorded. Increasing the heat sink temperature to $90 \mathrm{~K}$ increased the tuning range centered at $3.361 \mathrm{THz}$ to $20 \mathrm{GHz}$. Overall, emission frequencies centered at $3.307,3.335$, and $3.361 \mathrm{THz}$ with a continuous tuning range of 2,7 , and $20 \mathrm{GHz}$, and SMSR greater than 11,19 , and $25 \mathrm{~dB}$ respectively, were recorded. The gaps in the spectral coverage centered at 3.335 $\mathrm{THz}$ are due to atmospheric absorption. The slightly lower SMSR at $3.307 \mathrm{THz}$ and $3.335 \mathrm{THz}$ is due to the discrete mode hops. Nevertheless, an improvement in the SMSR of the emission frequencies centered at $3.361 \mathrm{THz}$ was observed due to the additive Vernier selection. The SMSR at this frequency was calculated to be in the range $25-40 \mathrm{~dB}$, and is higher than those reported from devices based on multiplicative Vernier selection, such as end-fire coupled cavities. We note that the frequency tuning in these devices could be expanded further by increasing the drive currents across the entire dynamic range of operation and also through localized Joule heating arising from quasi-direct current pulses (with pulse duty cycles $>50 \%$ ). However, these approaches were not pursued here, in order to minimize damage to the devices. The tuning performance could be further improved through facet coating.

Our approach enables a lookup table of QCL emission frequencies as a function of $I_{\mathrm{QCL}, 1}$ and $I_{\mathrm{QCL}, 2}$ to be developed, which can be used to programmatically tune the emission 


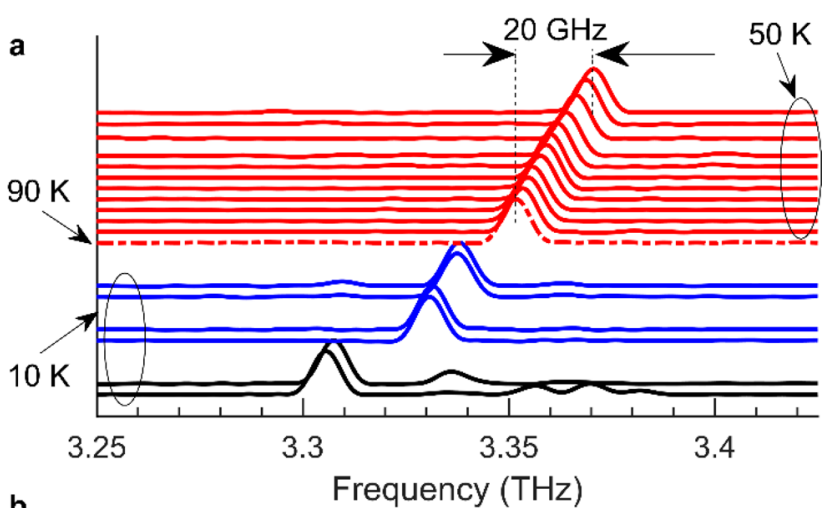

b

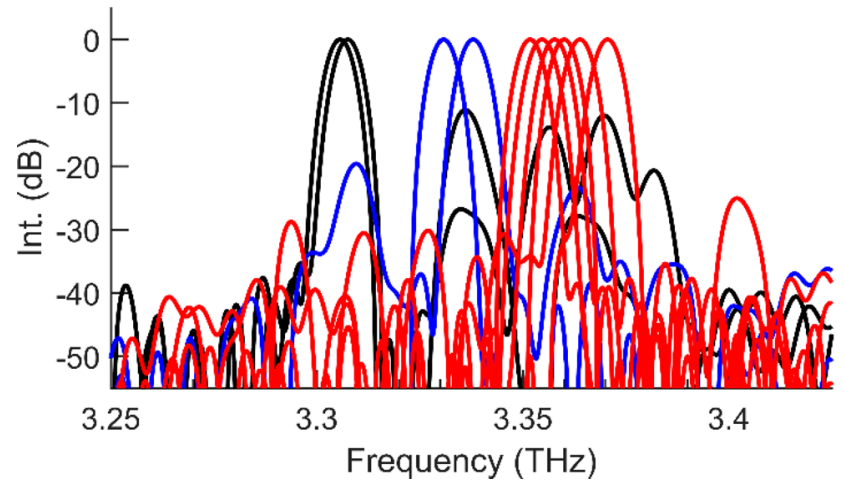

Figure 8. Experimentally recorded frequency tuning and SMSR. (a) Representative spectral coverage from the device obtained by varying $I_{\mathrm{QCL}, 1}, I_{\mathrm{QCL}, 2}$, and $T_{\mathrm{HS}}=10 \mathrm{~K}$ (black and blue), $50 \mathrm{~K}$ (red), and $90 \mathrm{~K}$ (red dashed). Spectra are offset, arranged in ascending order of emission frequencies, and continuous tunable frequency bands are color coded for clarity. (b) SMSR of the emission frequencies. A continuous frequency tuning of $20 \mathrm{GHz}$ from 3.351 to $3.371 \mathrm{THz}$ is measured with an SMSR of $>25 \mathrm{~dB}$.

frequency. Moreover, this scheme allows a fast frequency tuning of $\mathrm{THz}$ QCLs. Such a system can find application in both spectroscopy and interferometric applications, which require continuous tuning over a narrow frequency range (several $\mathrm{GHz}$ ), and in multifrequency imaging which require discrete frequency tuning (tens of $\mathrm{GHz}$ ).

\section{CONCLUSIONS}

In conclusion, the electrically controlled continuous frequency tuning range achievable in $\mathrm{DM} \mathrm{THz}$ QCLs has been increased by coupling two QCLs laterally along the length of the cavity to form a Y-branched coupler with integrated PL structures. One of the two PLs was optimized to emit a single frequency, whereas the other PL was optimized for discrete mode hops. Frequency tuning is controlled through both Stark shift of the gain and cavity pulling in the coupled device. The power distribution in the device was verified through a measurement of the $\mathrm{THz}$ output power emitted from both facets and the resulting asymmetry in the measured power from the Ybranches. Both continuous and discrete frequency tuning were observed from both QCLs when they operated alone at a heat sink temperature of $10 \mathrm{~K}$. However, a fast continuous tuning of $19 \mathrm{GHz}$ centered at $\sim 3.361 \mathrm{THz}$ was recorded with peak output powers in the range $\sim 4.2-4.8 \mathrm{~mW}$, when both QCLs were operated simultaneously at a constant heat sink temperature of $50 \mathrm{~K}$. Whereas the frequency tuning range reported here is less than that of $30 \mathrm{GHz}$ reported from a $\mathrm{PhC}$ $\mathrm{THz}$ QCL, ${ }^{15}$ unlike the PhC THz QCL our frequency tuning is achieved with a variation of output power variation of only $\sim 12.5 \%$. A variation of the current amplitudes in both QCLs as well the heat sink temperature allows emission at three frequencies with a maximum continuous tuning of $20 \mathrm{GHz}$ at $3.361 \mathrm{THz}$. Our approach allows a programmable and tunable source of $\mathrm{THz}$ radiation. These Y-branched DM THz QCLs can be optimized further to obtain both wideband discrete tuning, and narrowband continuous tuning. The far-field beam profile and the collection efficiency can be further improved by integrating diffraction gratings, ${ }^{29}$ and polarization of the radiation can be tuned by integrating an antenna array. ${ }^{30}$

\section{ASSOCIATED CONTENT}

S Supporting Information

The Supporting Information is available free of charge on the ACS Publications website at DOI: 10.1021/acsphotonics.8b00251.

Information showing the optical modes at the metallized and nonmetallized waveguide cross sections forming the photonic lattice calculated using finite element modeling, and the transfer matrix modeling of the photonic lattice (PDF).

\section{AUTHOR INFORMATION}

\section{Corresponding Author}

*E-mail: i.kundu@leeds.ac.uk.

ORCID $\odot$

Iman Kundu: 0000-0002-3564-1903

\section{Author Contributions}

I.K. conceived and conducted the experiments and simulations. I.K. and M.C.R. fabricated the devices. Y.H. designed the active region of the THz QCLs. E.H.L. and L.H.L. grew the $\mathrm{THz}$ QCL wafers. P.D., A.V., and J.R.F. analyzed the results. I.K. wrote the manuscript with contributions from all authors. All authors have given approval to the final version of the manuscript.

Notes

The authors declare no competing financial interest.

\section{ACKNOWLEDGMENTS}

This work was supported by the Engineering and Physical Sciences Research Council, U.K. (COTS and Hyper-Terahertz Programmes EP/J017671/1 and EP/P021859/1, respectively), UK Centre for Earth Observation Instrumentation (Contract RP10G0435A03), and European Space Agency (GSTP Contract 4000114487/15/NL/AF). E.H.L. and A.G.D. are grateful for support from the Royal Society (Wolfson Research Merit award WM150029). We thank L. Chen for his assistance during the fabrication. Research data associated with this paper are openly available from The University of Leeds data repository: http://doi.org/10.5518/332.

\section{REFERENCES}

(1) Köhler, R.; Tredicucci, A.; Beltram, F.; Beere, H. E.; Linfield, E. H.; Davies, A. G.; Ritchie, D. A.; Iotti, R. C.; Rossi, F. Terahertz Semiconductor-Heterostructure Laser. Nature 2002, 417 (6885), $156-159$.

(2) Hübers, H.-W.; Pavlov, S. G.; Richter, H.; Semenov, A. D.; Mahler, L.; Tredicucci, A.; Beere, H. E.; Ritchie, D. A. HighResolution Gas Phase Spectroscopy with a Distributed Feedback Terahertz Quantum Cascade Laser. Appl. Phys. Lett. 2006, 89 (6), 061115 . 
(3) Dean, P.; Valavanis, A.; Keeley, J.; Bertling, K.; Lim, Y. L.; Alhathlool, R.; Chowdhury, S.; Taimre, T.; Li, L. H.; Indjin, D. Coherent Three-Dimensional Terahertz Imaging through Self-Mixing in a Quantum Cascade Laser. Appl. Phys. Lett. 2013, 103 (18), 181112.

(4) Hubers, H. W. Terahertz Heterodyne Receivers. IEEE J. Sel. Top. Quantum Electron. 2008, 14 (2), 378-391.

(5) Williams, B. S. Terahertz Quantum-Cascade Lasers. Nat. Photonics 2007, 1 (9), 517-525.

(6) Vitiello, M. S.; Tredicucci, A. Tunable Emission in $\mathrm{THz}$ Quantum Cascade Lasers. IEEE Trans. Terahertz Sci. Technol. 2011, 1 (1), 76-84.

(7) Kundu, I.; Dean, P.; Valavanis, A.; Chen, L.; Li, L.; Cunningham, J. E.; Linfield, E. H.; Davies, A. G. Discrete Vernier Tuning in Terahertz Quantum Cascade Lasers Using Coupled Cavities. Opt. Express 2014, 22 (13), 16595-16605.

(8) Han, N.; de Geofroy, A.; Burghoff, D. P.; Chan, C. W. I.; Lee, A. W. M.; Reno, J. L.; Hu, Q. Broadband All-Electronically Tunable MEMS Terahertz Quantum Cascade Lasers. Opt. Lett. 2014, 39 (12), $3480-3483$.

(9) Castellano, F.; Bianchi, V.; Li, L.; Zhu, J.; Tredicucci, A.; Linfield, E. H.; Giles Davies, A.; Vitiello, M. S. Tuning a MicrocavityCoupled Terahertz Laser. Appl. Phys. Lett. 2015, 107 (26), 261108.

(10) Turčinková, D.; Amanti, M. I.; Castellano, F.; Beck, M.; Faist, J. Continuous Tuning of Terahertz Distributed Feedback Quantum Cascade Laser by Gas Condensation and Dielectric Deposition. Appl. Phys. Lett. 2013, 102 (18), 181113.

(11) Turčinková, D.; Amanti, M. I.; Scalari, G.; Beck, M.; Faist, J. Electrically Tunable Terahertz Quantum Cascade Lasers Based on a Two-Sections Interdigitated Distributed Feedback Cavity. Appl. Phys. Lett. 2015, 106 (13), 131107.

(12) Kundu, I.; Dean, P.; Valavanis, A.; Chen, L.; Li, L.; Cunningham, J. E.; Linfield, E. H.; Davies, A. G. Quasi-Continuous Frequency Tunable Terahertz Quantum Cascade Lasers with Coupled Cavity and Integrated Photonic Lattice. Opt. Express 2017, 25 (1), 486-496.

(13) Hempel, M.; Röben, B.; Niehle, M.; Schrottke, L.; Trampert, A.; Grahn, H. T. Continuous Tuning of Two-Section, Single-Mode Terahertz Quantum-Cascade Lasers by Fiber-Coupled, near-Infrared Illumination. AIP Adv. 2017, 7 (5), 055201.

(14) Wu, C.; Jin, Y.; Reno, J. L.; Kumar, S. Large Static Tuning of Narrow-Beam Terahertz Plasmonic Lasers Operating at $78 \mathrm{~K}$. APL Photonics 2017, 2 (2), 026101.

(15) Zhang, H.; Scalari, G.; Faist, J.; Dunbar, L. A.; Houdré, R. Design and Fabrication Technology for High Performance Electrical Pumped Terahertz Photonic Crystal Band Edge Lasers with Complete Photonic Band Gap. J. Appl. Phys. 2010, 108 (9), 093104.

(16) Coldren, L. A. Monolithic Tunable Diode Lasers. IEEE J. Sel. Top. Quantum Electron. 2000, 6 (6), 988-999.

(17) Li, H.; Manceau, J. M.; Andronico, A.; Jagtap, V.; Sirtori, C.; Li, L. H.; Linfield, E. H.; Davies, A. G.; Barbieri, S. Coupled-Cavity Terahertz Quantum Cascade Lasers for Single Mode Operation. Appl. Phys. Lett. 2014, 104 (24), 241102.

(18) Wesström, J.-O.; Sarlet, G.; Hammerfeldt, S.; Lundqvist, L.; Szabo, P.; Rigole, P.-J. State-of-the-Art Performance of Widely Tunable Modulated Grating Y-Branch Lasers. Optical Fiber Communication Conference, Technical Digest (CD); Optical Society of America: Los Angeles, United States, 2004; p TuE2.

(19) Kundu, I.; Dean, P.; Valavanis, A.; Li, L.; Han, Y.; Linfield, E. H.; Davies, A. G. Frequency Tunability and Spectral Control in Terahertz Quantum Cascade Lasers with Phase Adjusted Finite Defect Site Photonic Lattice. IEEE Trans. Terahertz Sci. Technol. 2017, 7 (4), 360-367.

(20) Young, J. S.; Kozlowski, D. A.; England, J. M. C.; Plumb, R. G. S. Spectral Perturbation and Mode Suppression in $1.3 \mathrm{Mm}$ FabryPerot Lasers. Electron. Lett. 1995, 31 (4), 290-291.

(21) Kozlowski, D. A.; Young, J. S.; England, J. M. C.; Plumb, R. G. S. Singlemode $1.3 \mathrm{Mm}$ Fabry-Perot Lasers by Mode Suppression. Electron. Lett. 1995, 31 (8), 648-650.
(22) Chassagneux, Y.; Colombelli, R.; Maineult, W.; Barbieri, S.; Beere, H. E.; Ritchie, D. A.; Khanna, S. P.; Linfield, E. H.; Davies, A. G. Electrically Pumped Photonic-Crystal Terahertz Lasers Controlled by Boundary Conditions. Nature 2009, 457 (7226), 174-178.

(23) Ye, L.; Zhang, Y.; Xu, R.; Lin, W. A Terahertz Broadband $3 \mathrm{~dB}$ Directional Coupler Based on Bridged PPDW. Opt. Express 2011, 19 (20), 18910-18916.

(24) Grover, R. Indium Phosphide Based Optical Micro-Ring Resonators; University of Maryland: College Park, 2003.

(25) Faist, J. Quantum Cascade Lasers, 1st ed.; Oxford University Press, 2013.

(26) Yariv, A. Quantum Electronics, 3rd ed.; John Wiley \& Sons, 1988.

(27) Wienold, M.; Schrottke, L.; Giehler, M.; Hey, R.; Anders, W.; Grahn, H. T. Low-Voltage Terahertz Quantum-Cascade Lasers Based on LO-Phonon-Assisted Interminiband Transitions. Electron. Lett. 2009, 45 (20), 1030-1031.

(28) HITRAN online. Water Vapour Absorption Spectra at Room Temperature; http://hitran.org/.

(29) Zhu, H.; Wang, F.; Yan, Q.; Yu, C.; Chen, J.; Xu, G.; He, L.; Li, L.; Chen, L.; Davies, A. G.; et al. Terahertz Master-Oscillator PowerAmplifier Quantum Cascade Lasers. Appl. Phys. Lett. 2016, 109 (23), 231105.

(30) Liang, G.; Zeng, Y.; Hu, X.; Yu, H.; Liang, H.; Zhang, Y.; Li, L.; Davies, A. G.; Linfield, E. H.; Wang, Q. J. Monolithic Semiconductor Lasers with Dynamically Tunable Linear-to-Circular Polarization. ACS Photonics 2017, 4 (3), 517. 